\title{
Microscopic Examinations of the Elements on the Behaviour of Group of Fellow Men using Fuzzy Graph
}

\section{P. Sinthamani}

\begin{abstract}
In this work, the behavior of an individual of a group to his fellow men. In a group, if we consider the relationship between people, we note the following possibilities. Any two of them may like each other or dislikes each other or indifferent to each other. It can be also checked originally to describe the way psychological consistency is obtained. And signed degree vertex evolves interesting results in new parameters of Balanced signed fuzzy graph and unbalanced signed fuzzy graph also we obtained some properties over it.
\end{abstract}

Keywords: Signed graph, Balanced Signed Graph.

\section{INTRODUCTION}

In connection with the problems in Social psychology, Cartwright and Harary [1] introduced the notion of signed graph. Here, nodes represent persons and the edges represent symmetric dyadic relations among them. The basic frame work for doing this is to understand the tension between these two forces, and the notion of structural balance. Afterward Zaslavsky [3] and many other authors established sequential results. It has important in the literature, due to its extensive use in the field of modeling; a variety of Socio-psychological processes and also it has various interesting connections with much classical mathematical analysis. It is clear that in real life many problems bear imprecise information, for model those problems introduced the concept of fuzzy set, which moderate many areas of Science and technology was introduced by Zadeh [5]. The concept of fuzzy graph was introduced by Rosenfeld [2].

In this article, we have introduced Fuzzy signed graphs and investigated a few of their properties. We have used the standard definitions and terminologies in this paper.

\section{PRELIMINARIES}

\subsection{Definition}

If each edge of the path of a graph has either a positive or negative sign, it is called a signed fuzzy graph.

Revised Manuscript Received on February 05, 2020.

* Correspondence Author

P. Sinthamani, Department of Mathematics, C. Kandaswami Naidu College for Women, Cuddalore-607 001, Tamil Nadu, India, psinthamani88@gmail.com.

(C) The Authors. Published by Blue Eyes Intelligence Engineering and Sciences Publication (BEIESP). This is an open access article under the CC BY-NC-ND license (http://creativecommons.org/licenses/by-nc-nd/4.0/)

\section{Example 2.1}
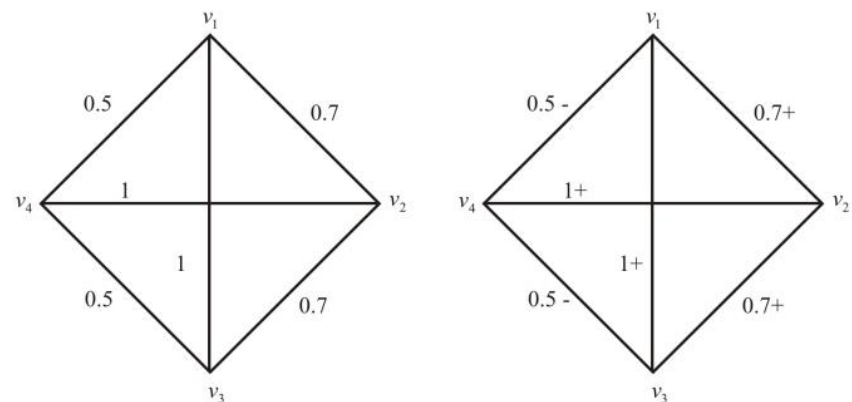

Fig.1. Fuzzy graph and its signed graph.

In this fuzzy graph we assume $\alpha=0.6, v_{1} v_{2}, v_{2} v_{3}, v_{1} v_{3}, v_{2} v_{4}$ are contained in the four edges of the $\alpha$-cut set. We have assigned positive sign to these edges and negative sign for the remaining.

\subsection{Definition}

If $d^{+}(v)=d^{-}(v)$ is true for every vertex $v$ of $D$, the directed graph $D$ is said to be a balanced fuzzy digraph or an isograph or a pseudo symmetric digraph.

The number of arcs that terminate at $v$ is the In-degree of $v$.

The number of arcs of the fuzzy graph $D$ is the Out-degree of a vertex $V$ that initiate at $V$.

Example 2.2

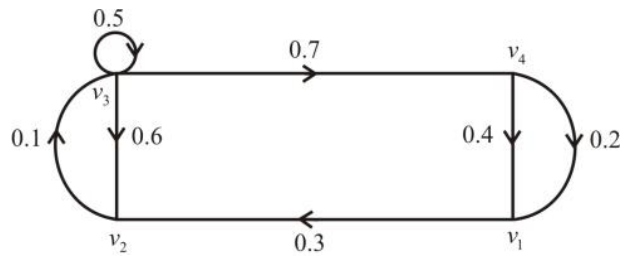

Fig.2. Balanced Fuzzy Digraph.

In this fuzzy graph, $d^{+}(v)$ denotes out-degree of $v$ and $d^{-}(v)$ denotes in-degree of $v$.

Here,

$$
\begin{aligned}
d^{+}\left(v_{1}\right) & =0.3 \\
d^{+}\left(v_{2}\right) & =0.1 \\
& d^{+}\left(v_{3}\right)=0.7+0.6+0.5=1.8 \\
d^{+}\left(v_{4}\right) & =0.4+0.2=0.6
\end{aligned}
$$

$\therefore$ Out degree of

$v_{i}=\left[d^{+}\left(v_{i}\right)\right]=2.8$ 


$$
\begin{aligned}
& d^{-}\left(v_{1}\right)=0.4+0.2=0.6 \\
& d^{-}\left(v_{2}\right)=0.6+0.3=0.9 \\
& d^{-}\left(v_{3}\right)=0.1+0.5=0.6 \\
& d^{-}\left(v_{4}\right)=0.7
\end{aligned}
$$

$\therefore$ In degree of $v_{i}=\left[d^{-}\left(v_{i}\right)\right]=2.8$

Here, $d^{+}(v)=d^{-}(v)$, therefore this fuzzy digraph is a balanced fuzzy digraph.

\subsection{Definition}

If every vertex has the same in-degree and out-degree as every other vertex, the balanced fuzzy digraph is said to be regular.

Example 2.3

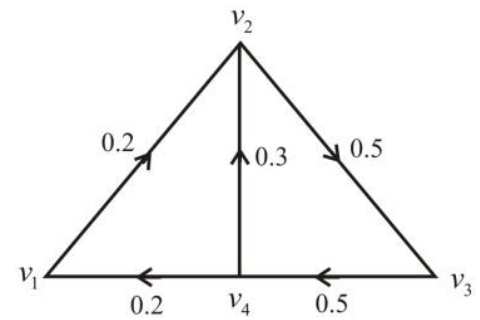

Fig.3. Regular Fuzzy Digraph.

In this fuzzy graph,

$$
\begin{aligned}
& d^{+}\left(v_{1}\right)=d^{-}\left(v_{1}\right)=0.2 \\
& d^{+}\left(v_{2}\right)=d^{-}\left(v_{2}\right)=0.5 \\
& d^{+}\left(v_{3}\right)=d^{-}\left(v_{3}\right)=0.5 \\
& d^{+}\left(v_{4}\right)=d^{-}\left(v_{4}\right)=0.5
\end{aligned}
$$

Therefore, every vertex has the same in-degree and out-degree.

\subsection{Definition}

The positive degree of any vertex $v$ is the sum of the membership values of all incident positive edge to $v$, that is, $\operatorname{deg}^{+}[\sigma(v)]=\sum_{\mu^{+}\left(v, v_{j}\right) \in E} \mu^{+}\left(v, v_{i}\right)$ and the negative degree of any vertex $\boldsymbol{v}$ is the sum of the membership values of all incident negative edge to $v$, that is, $\operatorname{deg}^{-}[\sigma(v)]=\sum_{\mu^{-}\left(v, v_{i}\right) \in E} \mu^{-}\left(v, v_{i}\right)$ and $s \operatorname{deg}(v)$ known as signed degree of any vertex $v$ is difference between $\operatorname{deg}^{+}[\sigma(v)]$ and $\operatorname{deg}^{-}[\sigma(v)]$ and that is, $s \operatorname{deg}[\sigma(v)]=\left|\operatorname{deg}^{+}[\sigma(v)]-\operatorname{deg}^{-}[\sigma(v)]\right|$ and the degree of $v$ is $\operatorname{deg}[\sigma(v)]=\operatorname{deg}^{+}[\sigma(v)]+\operatorname{deg}^{-}[\sigma(v)]$.

\section{Example 2.4}

The following is an example to calculate sign degree of all vertex of a fuzzy signed graph.

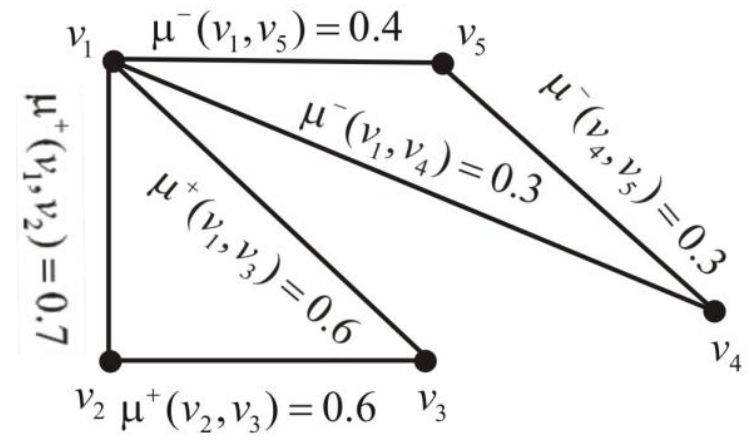

Fig.4. Signed degree of Fuzzy Graph. $\operatorname{deg}[\sigma(u)]=\operatorname{deg}^{+}[\sigma(u)]+\operatorname{deg}^{-}[\sigma(u)]$

$$
\begin{aligned}
& \begin{aligned}
\therefore \operatorname{deg}\left[\sigma\left(v_{1}\right)\right] & =\operatorname{deg}^{+}\left[\sigma\left(v_{1}\right)\right]+\operatorname{deg}^{-}\left[\sigma\left(v_{1}\right)\right] \\
& =[0.7+0.6]+[0.4+0.3] \\
& =2.0
\end{aligned} \\
& \begin{aligned}
\operatorname{deg}\left[\sigma\left(v_{2}\right)\right]= & \operatorname{deg}^{+}\left[\sigma\left(v_{2}\right)\right]+\operatorname{deg}^{-}\left[\sigma\left(v_{2}\right)\right] \\
& =[0.7+0.6]+0 \\
& =1.3 \\
\operatorname{deg}\left[\sigma\left(v_{3}\right)\right]= & \operatorname{deg}^{+}\left[\sigma\left(v_{3}\right)\right]+\operatorname{deg}^{-}\left[\sigma\left(v_{3}\right)\right] \\
& =[0.6+0.6]+0 \\
& =1.3 \\
\operatorname{deg}\left[\sigma\left(v_{4}\right)\right]= & \operatorname{deg}^{+}\left[\sigma\left(v_{4}\right)\right]+\operatorname{deg}^{-}\left[\sigma\left(v_{4}\right)\right] \\
& =0+[0.3+0.3] \\
& =0.6 \\
\operatorname{deg}\left[\sigma\left(v_{5}\right)\right]= & \operatorname{deg}^{+}\left[\sigma\left(v_{5}\right)\right]+\operatorname{deg}^{-}\left[\sigma\left(v_{5}\right)\right] \\
& =0+[0.4+0.3] \\
& =0.7
\end{aligned}
\end{aligned}
$$

and

$$
\begin{aligned}
s \operatorname{deg}[\sigma(u)] & =\left|\operatorname{deg}^{+}[\sigma(u)]-\operatorname{deg}^{-}[\sigma(u)]\right| \\
\therefore s \operatorname{deg}\left[\sigma\left(v_{1}\right)\right] & =\left|\operatorname{deg}^{+}\left[\sigma\left(v_{1}\right)\right]-\operatorname{deg}^{-}\left[\sigma\left(v_{1}\right)\right]\right| \\
& =[0.7+0.6]-[0.4+0.3] \\
& =|1.3-0.7| \\
& =0.6 \\
s \operatorname{deg}\left[\sigma\left(v_{2}\right)\right] & =\left|\operatorname{deg}^{+}\left[\sigma\left(v_{2}\right)\right]-\operatorname{deg}^{-}\left[\sigma\left(v_{2}\right)\right]\right| \\
& =|[0.7+0.6]-0| \\
& =1.3 \\
s \operatorname{deg}\left[\sigma\left(v_{3}\right)\right] & =\left|\operatorname{deg}^{+}\left[\sigma\left(v_{3}\right)\right]-\operatorname{deg}^{-}\left[\sigma\left(v_{3}\right)\right]\right| \\
& =|[0.6+0.6]-0| \\
& =1.2 \\
s \operatorname{deg}\left[\sigma\left(v_{4}\right)\right] & =\left|\operatorname{deg}^{+}\left[\sigma\left(v_{4}\right)\right]-\operatorname{deg}^{-}\left[\sigma\left(v_{4}\right)\right]\right| \\
& =|0-[0.3+0.3]| \\
& =0.6 \\
s \operatorname{deg}\left[\sigma\left(v_{5}\right)\right] & =\left|\operatorname{deg}^{+}\left[\sigma\left(v_{5}\right)\right]+\operatorname{deg}^{-}\left[\sigma\left(v_{5}\right)\right]\right| \\
& =|0-[0.4+0.3]| \\
& =0.7
\end{aligned}
$$

\subsection{Definition}

A fuzzy signed graph is supposed to be balanced if the product of sign of all edges for each cycle is positive and sum of the membership value of all edges for each cycle of length ' $n$ ' is greater than or equal to $n / 2$.

\section{Example 2.5}

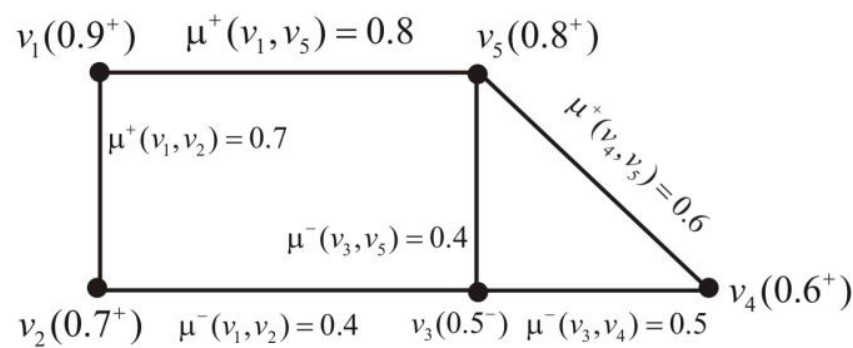

Fig.5. Balanced Fuzzy Signed Graph. 
In this fuzzy graph, each cycle $v_{1} v_{2} v_{3} v_{5}, v_{4} v_{3} v_{5}$ and $v_{1} v_{2} v_{3} v_{4} v_{5}$ is positive and sum of the membership of all edges for each cycle are 2.3, 1.5 and 3.0 respectively. Since for each cycle sum of the membership values of all edges becomes greater than or equal to $n / 2$, thus we can say that this fuzzy graph is balanced.

\subsection{Definition}

A signed fuzzy graph is balanced if every cycle has an even number of ' $-v e^{\prime}$ signs. Otherwise it is unbalanced signed fuzzy graph and a cycle has a positive if it has an even number of $-v e$ edges and a cycle has a negative if it has add number of $-v e$ edges.

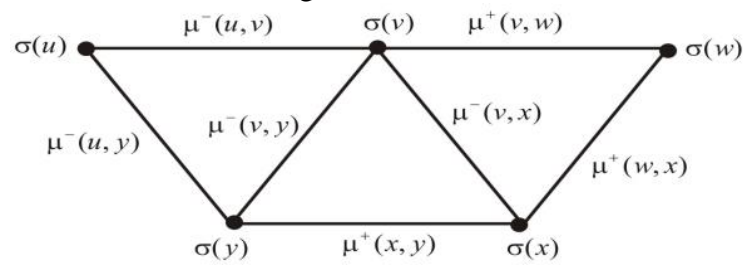

Fig.6. Signed Fuzzy Graph.

In this fuzzy graph,

\begin{tabular}{|l|c|c|c|}
\hline \multicolumn{1}{|c|}{ Cycle } & $\begin{array}{c}\text { No. of } \\
\text { negative } \\
\text { signs }\end{array}$ & $\begin{array}{c}\text { Balanced } / \\
\text { Unbalanced } \\
\text { fuzzy graph }\end{array}$ & $\begin{array}{c}\text { Positive } \\
\text { /Negative }\end{array}$ \\
\hline$\sigma(u) \sigma(v) \sigma(y)$ & 3 & Unbalanced & Negative \\
\hline$\sigma(v) \sigma(w) \sigma(x)$ & 1 & Unbalanced & Negative \\
\hline$\sigma(v) \sigma(x) \sigma(y)$ & 2 & Balanced & Positive \\
\hline$\sigma(u) \sigma(v) \sigma(w) \sigma(x) \sigma(y)$ & 2 & Balanced & Positive \\
\hline
\end{tabular}

\subsection{Definition}

A signed fuzzy graph $\mathrm{S}$ is said to be balanced if its vertex set can be partitioned into two subsets (one of which may be empty). So that any edge joining two vertices within the same subset is positive, while any edge joining two vertices in different subset is negative.

\section{Example 2.7}

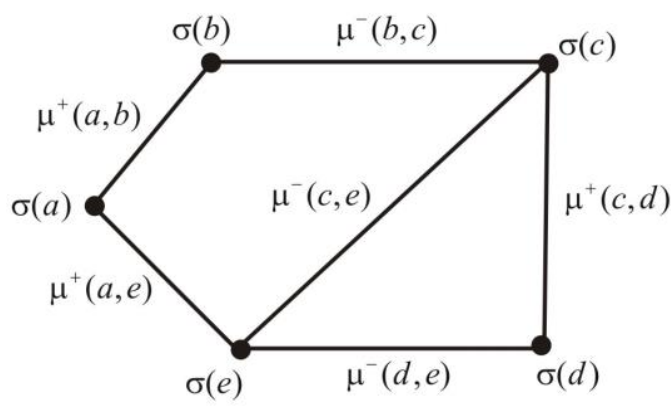

Fig.7. Signed Fuzzy Graph.

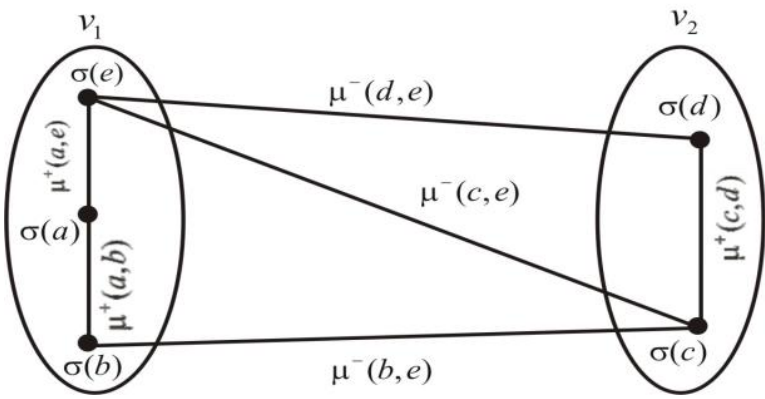

Fig.8. Balanced Signed Fuzzy graph.

\subsection{Theorem}

A Signed fuzzy graph is balanced if and only if for each pair of distinct pair of points $X$ and $Y$, all the paths joining $X$ and $Y$ have the same sign.

\subsection{Theorem}

A Signed fuzzy graph $G$ is called balanced if and only if its vertex set $V$ can be partitioned into two disjoint subsets namely $V_{1}$ and $V_{2}$, in such a way that each positive line of $G$ joints two points of the same subset and each negative line joints two points of different subsets.

\section{PROPOSED METHOD FOR THE NOTION OF STRUCTURAL BALANCED FUZZY GRAPH}

\subsection{Positive and Negative Relationship using Fuzzy graph}

If every edge has a positive or negative sign associated with it, it is called a signed fuzzy graph. Thus the four graphs of figures are signed fuzzy graphs. Let positive sign denotes friends and negative sign denotes enemies, then

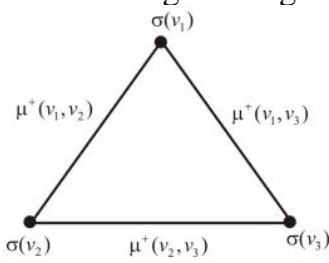

(1)

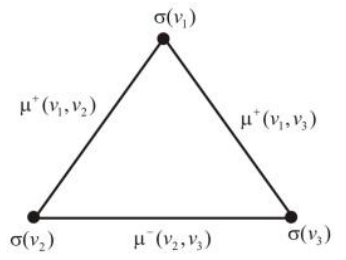

(3)

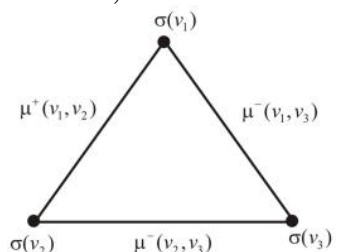

(2)

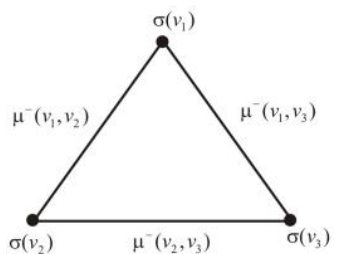

(4)
Fig.9. Signed Fuzzy graph.

In graph (1), $\sigma\left(v_{1}\right)$ is a friend of both $\sigma\left(v_{2}\right)$ and $\sigma\left(v_{3}\right)$ and $\sigma\left(v_{2}\right)$ and $\sigma\left(v_{3}\right)$ are also friend, this is balanced signed fuzzy graph [Because, three positive, the result will also be positive].

In graph (2), $\sigma\left(v_{1}\right)$ is a friend of $\sigma\left(v_{2}\right)$, but $\sigma\left(v_{1}\right)$ and $\sigma\left(v_{2}\right)$ are but jointly enemies of $\sigma\left(v_{3}\right)$, This is balanced sign fuzzy graph.

In graph (3), $\sigma\left(v_{1}\right)$ is a friend of both $\sigma\left(v_{2}\right)$ and $\sigma\left(v_{3}\right)$ but $\sigma\left(v_{2}\right)$ and $\sigma\left(v_{3}\right)$ are enemies, this is unbalanced fuzzy signed graph.

In graph (4), $\sigma\left(v_{1}\right)$ is a enemy of both $\sigma\left(v_{2}\right)$ and $\sigma\left(v_{3}\right)$, and $\sigma\left(v_{2}\right)$ and $\sigma\left(v_{3}\right)$ are not friends. This is also unbalanced fuzzy signed graph.

In this fuzzy graph there can be 8 relational configurations, relationships from $a$ to $d$ are balanced and those from $e$ to $h$ are unbalanced.

Balanced Fuzzy graph: 
a) $\sigma\left(v_{1}\right)+\sigma\left(v_{2}\right), \sigma\left(v_{1}\right)+\sigma\left(v_{3}\right), \sigma\left(v_{2}\right)+\sigma\left(v_{3}\right)$

b) $\sigma\left(v_{1}\right)-\sigma\left(v_{2}\right), \sigma\left(v_{1}\right)-\sigma\left(v_{3}\right), \sigma\left(v_{2}\right)+\sigma\left(v_{3}\right)$

c) $\sigma\left(v_{1}\right)-\sigma\left(v_{2}\right), \sigma\left(v_{1}\right)+\sigma\left(v_{3}\right), \sigma\left(v_{2}\right)-\sigma\left(v_{3}\right)$

d) $\sigma\left(v_{1}\right)+\sigma\left(v_{2}\right), \sigma\left(v_{1}\right)-\sigma\left(v_{3}\right), \sigma\left(v_{2}\right)-\sigma\left(v_{3}\right)$

Unbalanced Fuzzy graph:

e) $\sigma\left(v_{1}\right)+\sigma\left(v_{2}\right), \sigma\left(v_{1}\right)-\sigma\left(v_{3}\right), \sigma\left(v_{2}\right)+\sigma\left(v_{3}\right)$

f) $\sigma\left(v_{1}\right)+\sigma\left(v_{2}\right), \sigma\left(v_{1}\right)+\sigma\left(v_{3}\right), \sigma\left(v_{2}\right)-\sigma\left(v_{3}\right)$

g) $\sigma\left(v_{1}\right)-\sigma\left(v_{2}\right), \sigma\left(v_{1}\right)+\sigma\left(v_{3}\right), \sigma\left(v_{2}\right)+\sigma\left(v_{3}\right)$

h) $\sigma\left(v_{1}\right)-\sigma\left(v_{2}\right), \sigma\left(v_{1}\right)-\sigma\left(v_{3}\right), \sigma\left(v_{2}\right)-\sigma\left(v_{3}\right)$

Triangles with one or three ' + ' are referred to as balanced, because they are free of these sources of instability. Triangles with zero or two ' + ' are referred to as unbalanced.

Balanced theory is the triadic relationship between $\sigma\left(v_{1}\right), \sigma\left(v_{2}\right)$ and $\sigma\left(v_{3}\right)$. If $\sigma\left(v_{3}\right)$ likes $\sigma\left(v_{1}\right)$, then $\left[\sigma\left(v_{3}\right)+\sigma\left(v_{1}\right)\right]$, and $\sigma\left(v_{1}\right)$ states $\sigma\left(v_{2}\right)$ are great $\left[\sigma\left(v_{1}\right)+\sigma\left(v_{2}\right)\right]$, if he doesn't like $\sigma\left(v_{2}\right)$, then $\left[\sigma\left(v_{3}\right)-\sigma\left(v_{2}\right)\right]$ he feels uncomfortable and the relationship is imbalanced.

Therefore to restore the balance he will change his attitude into $\left[\sigma\left(v_{2}\right)+\sigma\left(v_{3}\right)\right]$ and return to configuration number 1 .

People don't relate any cognition in each other to find inconsistency. But may consider that an attitude change is likely to happen when they are aware of any inconsistency.

The first two fuzzy graphs represent normal behavior and are said to be balanced, while the last two fuzzy graphs represent unbalanced situation since it $\sigma\left(v_{1}\right)$ is a friend of both $\sigma\left(v_{2}\right)$ and $\sigma\left(v_{3}\right)$, and $\sigma\left(v_{2}\right)$ and $\sigma\left(v_{3}\right)$ are enemies, this creates a tension in the system and there is a similar tension when $\sigma\left(v_{2}\right)$ and $\sigma\left(v_{3}\right)$ have common enemy $\sigma\left(v_{1}\right)$, but are not friends of each other.

\subsection{Formulation of the Structural Balanced Problem}

A structural balanced problem can be modeled by a fuzzy graph where the nodes represent the various group of people and the edges represent the relationship among them. Thus the structural balanced problem results in a complete graph, that is, every node is connected to every other node by an edge with a sign associated with it.

Here a structural balanced problem for symmetric case at seven people is considered, where $\sigma\left(v_{1}\right), \sigma\left(v_{2}\right), \sigma\left(v_{3}\right), \sigma\left(v_{4}\right), \sigma\left(v_{5}\right), \sigma\left(v_{6}\right), \sigma\left(v_{7}\right)$ are the group of people. Here two cases will be shown, one for the balanced situation and another for unbalanced situation as shown below:

\section{Case I}

Representing a balance structural problem by using signed fuzzy graph. The relationship of the people is shown in Fig 10.

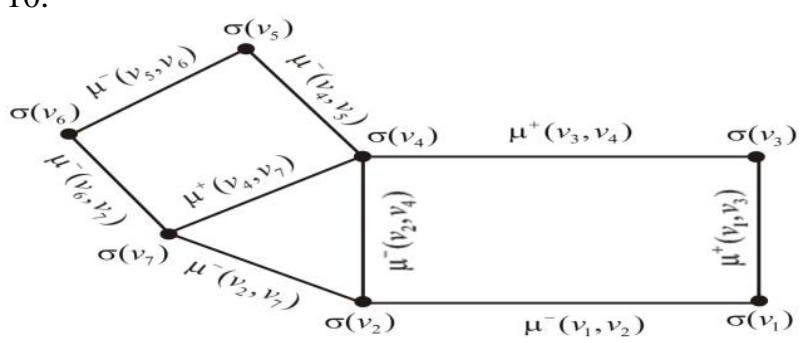

Fig.10. Balance network.

Here, vertices represent the groups of people and the edges joining the vertices represent a relationship between the

people. The positive $(+)$ sign indicates friends, and the negative (-) sign indicates enemies. Therefore, from the Theorem 2.8 and Theorem 2.9, it is found that the system represented by Fig.10 is a balanced. As vertex set can be partitioned into two disjoint subsets and edges joining the vertices of the same subset are positive and the edges joining the vertices of two different subsets are negative.

Here the vertices $\sigma\left(v_{1}\right), \sigma\left(v_{3}\right), \sigma\left(v_{4}\right), \sigma\left(v_{7}\right)$ can be put in one set as $X=\left\{\sigma\left(v_{1}\right), \sigma\left(v_{3}\right), \sigma\left(v_{4}\right), \sigma\left(v_{7}\right)\right\}$, and the vertices $\sigma\left(v_{2}\right), \sigma\left(v_{5}\right), \sigma\left(v_{6}\right)$ can be put another set as $Y=\left\{\sigma\left(v_{2}\right), \sigma\left(v_{5}\right), \sigma\left(v_{6}\right)\right\}$, such that every edges between $X=\left\{\sigma\left(v_{1}\right), \sigma\left(v_{3}\right), \sigma\left(v_{4}\right), \sigma\left(v_{7}\right)\right\}$, is positive and the edges from the vertex set $Y=\left\{\sigma\left(v_{2}\right), \sigma\left(v_{5}\right), \sigma\left(v_{6}\right)\right\}$, to any vertex of $X=\left\{\sigma\left(v_{1}\right), \sigma\left(v_{3}\right), \sigma\left(v_{4}\right), \sigma\left(v_{7}\right)\right\}$, are negative. Hence, it is balanced.

\section{Case II}

Representing an unbalance structural problem by using signed fuzzy graph. The relationship of the people is shown in Fig 11.

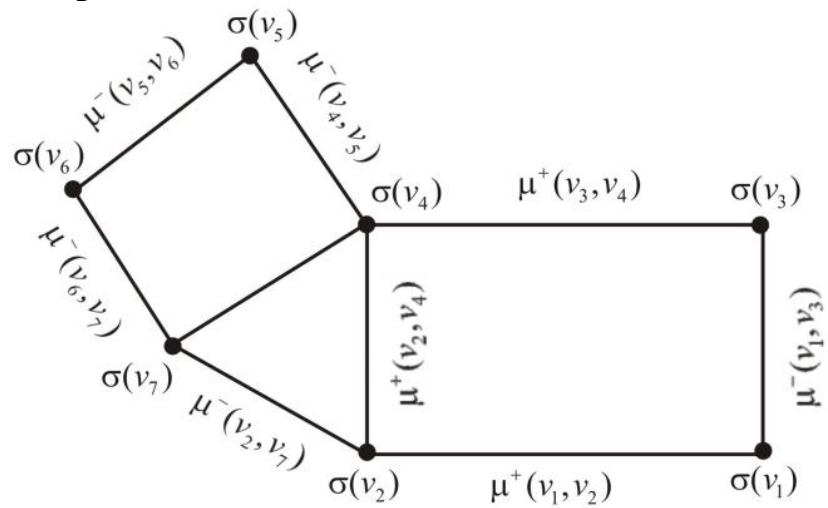

Fig.11. Unbalanced Network

Here the vertices $\sigma\left(v_{1}\right), \sigma\left(v_{3}\right), \sigma\left(v_{4}\right), \sigma\left(v_{7}\right)$ can be put in one set such as $X=\left\{\sigma\left(v_{1}\right), \sigma\left(v_{3}\right), \sigma\left(v_{4}\right), \sigma\left(v_{7}\right)\right\}$, and the vertices $\sigma\left(v_{2}\right), \sigma\left(v_{5}\right), \sigma\left(v_{6}\right)$ can be put in another set as $Y=\left\{\sigma\left(v_{2}\right), \sigma\left(v_{5}\right), \sigma\left(v_{6}\right)\right\}$. The above signed fuzzy graph in fig. 11 is unbalanced. That is, the set of vertices set $X$ cannot be partitioned into two disjoint subsets. Also there are two negative cycles which means that the above fuzzy graph representing the structural problem is unbalanced.

\subsection{The degree of unbalanced sign of a fuzzy graph}

The degree of balance of a fuzzy graph $G$ to be the ratio of the positive cycles of $G$ to the real number of cycles in $G$. This balanced index obviously lies between 0 and 1 .

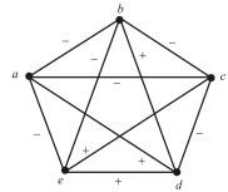

$G_{1}$

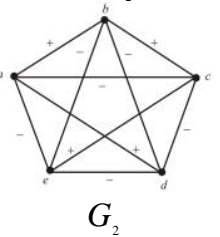

$G_{2}$

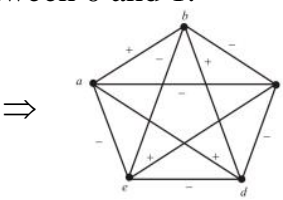$$
\text { (1) }
$$

Fig.12. Signed fuzzy graph. 
$G_{1}$ has six negative triangles $a b c$, ade, bcd, bce, bde, cde and has four positive triangles abe, acd, abd, ace.

$G_{2}$ has four negative triangles namely $a b c, a b d, b c e$, bde and has six positive triangles ade,bcd,cde, abe, acd, ace.

The degree of balance of $G_{1}$ is less than the degree of balance of $G_{2}$.

However in order to get a balanced graph from $G_{1}$, we have to change the sign of only two edges which is $b c$ and de and similarly to make $G_{2}$ balanced we have to change the signs of two edges as follows $b c$ and $b d$. From this point of view both $G_{1}$ and $G_{2}$ are equally unbalanced.

\section{Theorem 3.1}

Any fuzzy signed graph $G$, the sum of signed degrees of vertices of even degree is equal to twice the signed degree of membership of all the edges and the difference of the sum of signed degrees of vertices of odd degree.

\section{Proof}

Let $G:(\sigma, \mu)$ is a signed fuzzy graph. Consider even vertices $\sigma\left(v_{1}\right), \sigma\left(v_{2}\right), \sigma\left(v_{3}\right), \sigma\left(v_{4}\right), \sigma\left(v_{5}\right), \sigma\left(v_{6}\right), \sigma\left(v_{7}\right)$ of fuzzy signed graph $G:(\sigma, \mu)$.

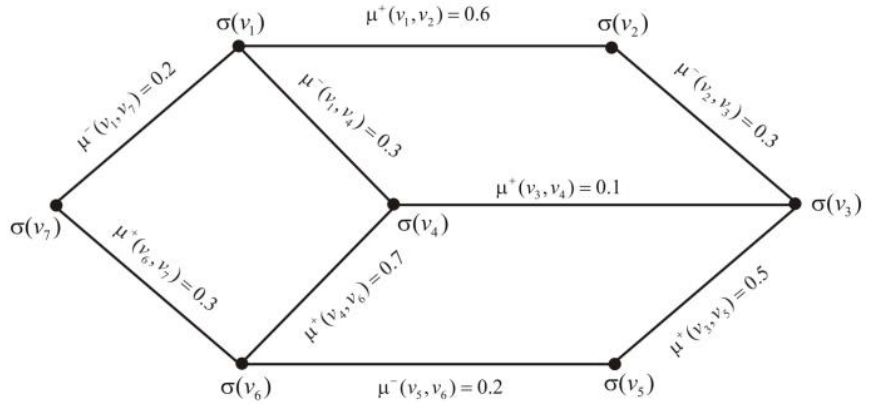

Fig.13. Signed Fuzzy Graph.

If the membership grades of edges which are incident on any signed degree of vertex $\sigma\left(v_{i}\right)$ are added, then the signed degree vertex is equal to

$s \operatorname{deg}[\sigma(u)]=\left|\operatorname{deg}^{+}[\sigma(u)]-\operatorname{deg}^{-}[\sigma(u)]\right|$

corresponding membership values of vertices vary,

$s \operatorname{deg}\left[\sigma\left(v_{1}\right)\right]=0.6-[0.2+0.3]=0.1$

$s \operatorname{deg}\left[\sigma\left(v_{2}\right)\right]=0.6-0.3=0.3$

$s \operatorname{deg}\left[\sigma\left(v_{3}\right)\right]=[0.5+0.1]-0.3=0.3$

$s \operatorname{deg}\left[\sigma\left(v_{4}\right)\right]=[0.7+0.1]-0.3=0.5$

$s \operatorname{deg}\left[\sigma\left(v_{5}\right)\right]=0.5-0.2=0.3$

$s \operatorname{deg}\left[\sigma\left(v_{6}\right)\right]=[0.7+0.3]-0.2=0.8$

$s \operatorname{deg}\left[\sigma\left(v_{7}\right)\right]=0.3-0.2=0.1$

If the membership grades of edges are added, then find the sign degree of edges,

$$
\begin{aligned}
\sum_{i=1}^{7} \mu\left(u_{i}, v_{i+1}\right)=\mid \operatorname{deg}^{+} & {[\sigma(u)]-\operatorname{deg}^{-}[\sigma(u)] \mid } \\
=[0.6+0.1+0.5 & +0.7+0.3]-[0.2+0.3+0.3+0.2] \\
= & 2.2-1 \\
= & 1.2
\end{aligned}
$$$$
\therefore \quad \sum_{i=1}^{7} \mu\left(u_{i}, v_{i+1}\right)=1.2
$$

$\sum_{i=1}^{n} s \operatorname{deg}\left[\sigma\left(v_{i}\right)\right]=$ twice the sum of signed degree of membership of $\left(u_{i}, v_{i+1}\right)$.

$$
\therefore \sum_{i=1}^{n} s \cdot \operatorname{deg}\left[\sigma\left(v_{i}\right)\right]=2 \sum_{i=1}^{n} \mu\left(u_{i}, v_{i+1}\right)
$$

But, here s.deg[ $\left.\sigma\left(v_{i}\right)\right]$ has been splited into two parts.

i.e., $\sum_{i=1}^{K} s . \operatorname{deg} v_{i}+\sum_{i=1}^{n} s . \operatorname{deg} w_{k} 2 \sum_{i=1}^{n} \mu\left(u_{i}, v_{i+1}\right)$

Here, $\sum_{i=1}^{K} s \cdot \operatorname{deg} v_{i}$ is the sum over even degree vertices (i.e., $\left.\sigma\left(v_{2}\right), \sigma\left(v_{4}\right), \sigma\left(v_{6}\right)\right)$

$\sum_{i=1}^{K} s \cdot \operatorname{deg} v_{i}=s \cdot \operatorname{deg}\left[\sigma\left(v_{2}\right)\right]+s \cdot \operatorname{deg}\left[\sigma\left(v_{4}\right)\right]+s \cdot \operatorname{deg}\left[\sigma\left(v_{6}\right)\right]$

$$
=0.3+0.5+0.8
$$

$\sum_{i=1}^{K} s . \operatorname{deg} v_{i}=1.6$

$\sum_{i=1}^{n} s . \operatorname{deg} w_{K} \quad$ is the sum over odd degree vertices (i.e., $\left.\sigma\left(v_{1}\right), \sigma\left(v_{3}\right), \sigma\left(v_{5}\right), \sigma\left(v_{7}\right)\right)$

$\sum_{i=1}^{n} s \cdot \operatorname{deg} w_{k}=s \cdot \operatorname{deg}\left[\sigma\left(v_{1}\right)\right]+s \cdot \operatorname{deg}\left[\sigma\left(v_{3}\right)\right]+s \cdot \operatorname{deg}\left[\sigma\left(v_{5}\right)\right]+s \cdot \operatorname{deg}\left[\sigma\left(v_{7}\right)\right]$

$$
=0.1+0.3+0.3+0.1=0.8
$$

$\therefore \sum_{i=1}^{n} s \cdot \operatorname{deg} w_{K}=0.8$

The sum of signed degrees of vertices of even degree is equal to twice the degree of membership of all the edges and the difference of the sum of signed degrees of vertices of odd degree.

$$
\text { (i.e) } \begin{aligned}
\sum_{i=1}^{K} s . \operatorname{deg} v_{i} & =2 \sum_{i=1}^{n} \mu\left(u_{i}, v_{i+1}\right)-\sum_{i=1}^{n} s . \operatorname{deg} w_{K} \\
1.6 & =2(1.2)-0.8 \\
& =2.4-0.8 \\
1.6 & =1.6
\end{aligned}
$$

Hence the theorem.

\section{Theorem 3.2}

For any signed fuzzy graph $G:(\sigma, \mu)$ without isolated vertices, $\quad \sum_{v \in V} \operatorname{deg}[\sigma(v)]+2 n_{0}=2 \Sigma \mu\left(v_{i}, v_{i+1}\right) \quad$ and $\sum_{v \in V}|\operatorname{s.deg}[\sigma(v)]| \leq 2 \Sigma \mu\left(v_{i}, v_{i}+1\right)$.

\section{Proof}

A signed fuzzy graph is one in which every edge has a positive or negative sign associated with it.

Let positive sign denote friends and negative sign denotes enemies.

Given signed fuzzy graph $G:(\sigma, \mu)$ without isolated vertices. First each

$$
\operatorname{deg}[\sigma(v)]=\operatorname{deg}^{+}[\sigma(v)]+\operatorname{deg}^{-}[\sigma(v)]
$$

In a signed fuzzy graph $G:(\sigma, \mu)$ with $|V|=\sigma(v)$, $|E|=\Sigma \mu\left(v_{i}, v_{i+1}\right)$, we denote $\mu^{+}\left(v_{i}, v_{i+1}\right)$ the number of positive edges of $G$ and $\mu^{-}\left(v_{i}, v_{i+1}\right)$ denote the number of negative edges of $G$ respectively. 
$\sigma\left(n_{0}\right)$ denote the number of vertices with zero signed fuzzy degrees. Since $G$ has no isolated vertices, $s . \sigma\left(n_{0}\right)=0$. Thus,

$$
\begin{aligned}
& \sum_{v \in V} \operatorname{deg}[\sigma(v)]+2 . \sigma\left(n_{0}\right)=\sum_{v \in V} \operatorname{deg}^{+}[\sigma(v)]+\operatorname{deg}^{-}[\sigma(v)]+2 \sigma\left(n_{0}\right) \\
& =2 \Sigma \mu^{+}\left(v_{i}, v_{i+1}\right)+2 \Sigma \mu^{-}\left(v_{i}, v_{i+1}\right)+2 \sigma\left(n_{0}\right) \\
& \therefore \sum_{v \in V} \operatorname{deg}[\sigma(v)]+2 . \sigma\left(n_{0}\right)=2 \Sigma \mu\left(v_{i}, v_{i+1}\right)+2 \sigma\left(n_{0}\right)
\end{aligned}
$$

\section{Example}

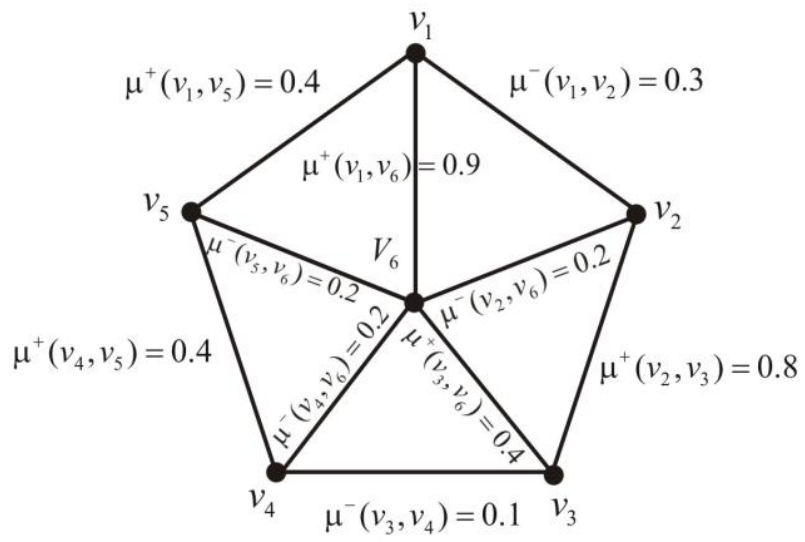

Fig.14. Fuzzy signed graph.

$\operatorname{deg}[\sigma(v)]=\operatorname{deg}^{+}[\sigma(v)]+\operatorname{deg}^{-}[\sigma(v)]$

$$
\begin{aligned}
& \operatorname{deg}\left[\sigma\left(v_{1}\right)\right]=0.9+0.4+0.3=1.6 \\
& \operatorname{deg}\left[\sigma\left(v_{2}\right)\right]=0.8+0.3+0.2=1.3 \\
& \operatorname{deg}\left[\sigma\left(v_{3}\right)\right]=0.8+0.4+0.1=1.3 \\
& \operatorname{deg}\left[\sigma\left(v_{4}\right)\right]=0.4+0.2+0.1=0.7 \\
& \operatorname{deg}\left[\sigma\left(v_{5}\right)\right]=0.4+0.4+0.2=1.0 \\
& \operatorname{deg}\left[\sigma\left(v_{6}\right)\right]=0.9+0.4+0.2+0.2+0.2=1.9
\end{aligned}
$$

Sum of degrees of all vertices,

$\operatorname{deg}[\sigma(v)]=1.6+1.3+1.3+0.7+1.0+1.9$

$\operatorname{deg}[\sigma(v)]=7.8$

In this fuzzy graph $\sigma\left(n_{0}\right)=0$

To find, $2 \Sigma \mu^{+}\left(v_{i}, v_{i+1}\right)$ and $2 \Sigma \mu^{-}\left(v_{i}, v_{i+1}\right)$

$$
\begin{gathered}
\therefore \mu^{+}\left(v_{i}, v_{i+1}\right)=0.4+0.4+0.8+0.4+0.9=2.9 \\
\mu^{-}\left(v_{i}, v_{i+1}\right)=0.3+0.1+0.2+0.2+0.2=1 \\
\therefore 2 \Sigma \mu\left(v_{i}, v_{i+1}\right)=2 \Sigma \mu^{+}\left(v_{i}, v_{i+1}\right)+2 \Sigma \mu^{-}\left(v_{i}, v_{i+1}\right) \\
=2(2.9)+2(1) \\
=5.8+2 \\
\therefore 2 \Sigma \mu\left(v_{i}, v_{i+1}\right)=7.8 \\
\therefore \sum_{v \in V} \operatorname{deg}[\sigma(v)]=2 \Sigma \mu\left(v_{i}, v_{i+1}\right) \\
7.8=7.8
\end{gathered}
$$

$\sum_{v \in V}|\operatorname{s.deg}[\sigma(v)]| \leq 2 \Sigma \mu\left(v_{i}, v_{i+1}\right)$

$|\operatorname{s.deg}[\sigma(v)]|=\left|\operatorname{deg}^{+}[\sigma(v)]-\operatorname{deg}^{-}[\sigma(v)]\right|$

$$
\begin{aligned}
& s . \operatorname{deg}\left[\sigma\left(v_{1}\right)\right]=0.9+0.4-0.3=1 \\
& s . \operatorname{deg}\left[\sigma\left(v_{2}\right)\right]=0.8-[0.3+0.2]=0.3 \\
& s . \operatorname{deg}\left[\sigma\left(v_{3}\right)\right]=0.8+0.4-0.1=1.1 \\
& s . \operatorname{deg}\left[\sigma\left(v_{4}\right)\right]=0.4-[0.2+0.1]=0.1 \\
& s . \operatorname{deg}\left[\sigma\left(v_{5}\right)\right]=0.4+0.4-0.2=0.6 \\
& s . \operatorname{deg}\left[\sigma\left(v_{6}\right)\right]=0.9+0.4-[0.2+0.2+0.2]=0.7
\end{aligned}
$$

$\therefore$ Sum of signed degrees of all vertices,

$$
\begin{gathered}
\text { |s.deg }[\sigma(v)]=1+0.3+1.1+0.1+0.6+0.7 \\
\quad=3.8 \\
\begin{array}{c}
\therefore \sum_{v \in V}|\operatorname{s.deg}[\sigma(v)]| \leq 2 \Sigma \mu\left(v_{i}, v_{i+1}\right) \\
3.8 \leq 7.8
\end{array}
\end{gathered}
$$

Hence proved.

\section{RESULT AND DISCUSSION}

\begin{tabular}{|l|l|l|l|l|}
\hline $\begin{array}{l}\text { S. } \\
\text { No. }\end{array}$ & People & $\begin{array}{c}\text { Relation } \\
\text { between people } \\
\text { of a group }\end{array}$ & Character & $\begin{array}{l}\text { Relation } \\
\text { between } \\
\text { people }\end{array}$ \\
\hline 1. & $\sigma\left(v_{1}\right)$ & $\sigma\left(v_{1}\right)-\sigma\left(v_{2}\right)$ & Negative & Enemy \\
\cline { 3 - 5 } & $\sigma\left(v_{1}\right)-\sigma\left(v_{3}\right)$ & Positive & Friend \\
\hline 2. & $\sigma\left(v_{2}\right)$ & $\sigma\left(v_{2}\right)-\sigma\left(v_{4}\right)$ & Negative & Enemy \\
\cline { 3 - 5 } & & $\sigma\left(v_{2}\right)-\sigma\left(v_{7}\right)$ & Negative & Enemy \\
\hline 3. & $\sigma\left(v_{3}\right)$ & $\sigma\left(v_{3}\right)-\sigma\left(v_{4}\right)$ & Positive & Friend \\
\hline 4. & $\sigma\left(v_{4}\right)$ & $\sigma\left(v_{4}\right)-\sigma\left(v_{5}\right)$ & Negative & Enemy \\
\cline { 3 - 5 } & & $\sigma\left(v_{4}\right)-\sigma\left(v_{7}\right)$ & Positive & Friend \\
\hline 5. & $\sigma\left(v_{5}\right)$ & $\sigma\left(v_{5}\right)-\sigma\left(v_{6}\right)$ & Negative & Enemy \\
\hline 6. & $\sigma\left(v_{6}\right)$ & $\sigma\left(v_{6}\right)-\sigma\left(v_{7}\right)$ & Negative & Enemy \\
\hline
\end{tabular}

It is clear that the structural problem at a balanced situation is smooth; this situation is used to have a sense of working well together, without tension to have good level of mutual awareness for a small group of people. On the other hand the structural problem at an unbalanced situation is compared to the balanced situation; this creates a tension in the system. Therefore to restore the balance he will change his attitude negative into positive. Though here comparatively considered less number of people, however the procedure can be generalized and applied to large number of people and to be checked whether attitude among the people are efficient or not. In a group consisting of persons with positive and negative attitudes the fuzzy signed graph place an important role to justify the decision taken. Instead of terms like and dislike for investigating the relationship between people of a group the model yields a respectable outcome in the future.

\section{CONCLUSION}

To study this type of problems, signed fuzzy graph, will be very useful. We use more general terms like positive relation and negative relation instead of terms like and dislike in investigating the relationship between people of a group. Thus the model makes maximum sense for a group of people small enough to have this level of mutual awareness. And also we analyzed the ideas on signed graph properties of fuzzy signed graph based on its degree sequence.

\section{REFERENCES}

1. Cartwright, D., and Harary, F., "Structural Balance: A Generalization of Heiders Theory.” Psych. Rev., 63 (1956): 277-293.

2. Rosenfeld, A., "Fuzzy Graphs." Fuzzy Sets and their Applications [Zadeh, L.A., Fu, K.S., and Shimura, M. (eds.)], New York: Academic Press, 1975: 75-95.

3. Zaslavsky, T., "Signed Graphs." Discrete Applies Mathematics, 4 (1982): 47-74. 
4. Nirmala, G., and Prabavathi, S., "Mathematical models in terms of balanced signed Fuzzy graphs with generalized modus ponens method." International Journal of Scientific and Research (IJSR), 4(7) (2015): 2415-2419.

5. Zadeh, L.A., "The Concept of a Linguistic and Application to Approximate reasoning-I.” Information Sci., 8 (1975): 199-249.

6. Bhattacharya, P., "Some remarks on fuzzy graphs." Pattern Recognition Lett., 6 (1991): 413-420.

7. Nirmala, G., and Sinthamani, P., "Fuzzy Regular Graph Properties with IF-THEN Rules." Aryabhatta Journal of Mathematics and Informatics, 7 (2015): 97-108.

8. Harary, F., "On the Notion of Balance of a Signed Graph." Michigan Math. J., 2 (1954): 143-146.

9. Zaslavsky, T., "Negative (and positive) circles in signed graphs: A problem Collection.” AKCE International Journal of Graphs and Combinatorics, 15 (2018): 31-48.

10. Nagoorgani, A., and Radha, K., "The degree of a vertex in some fuzzy graphs." International Journal of Algorithms, Computing and Mathematics, 2 (2009): 107-116.

11. Radha, K., and Rosemine, A., "Degree set of a fuzzy graph." International Journal of Mathematical Achieve, 6 (2015): 102-106.

12. Sunitha, M.S., and Sunil Mathew, "A survey of fuzzy graph theory." Annals of Pure and Applied Mathematics, 4 (2013): 92-110.

13. Vaishnaw, Y., and Sharma, S., "Some analogues results on fuzzy graphs." International Journal of Mathematics Sciences and Applications, 2 (2012): 535-539.

\section{AUTHOR PROFILE}

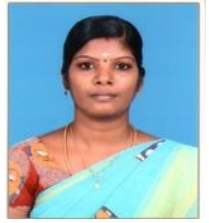

Dr.P.SINTHAMANI received her Master degree, Master of Philosophy and Doctor of Philosophy in Mathematics from Bharathidasan University, Trichy, Tamil Nadu. She is currently an Assistant Professor in the department of Mathematics at C.Kandaswami naidu college for women, Cuddalore, Tamil Nadu. Her areas of interest are Fuzzy Mathematics. 